\title{
Aortic plaque-dual therapy for a heterogeneous disease
}

Combination therapy with a statin and a bisphosphonate reduces atherosclerotic plaque in both the thoracic and abdominal aorta, whereas statin monotherapy is only effective in the thoracic aorta and a bisphosphonate alone only reduces plaque in the abdominal aorta. These findings from a study by Kawahara et al. highlight the differences in plaque composition between these two areas of the aorta.

Thoracic aortic plaques usually contain fatty streaks, whereas those in the abdominal aorta tend to be fibrous and calcified, which could explain the observed resistance of abdominal aortic plaques to statin therapy. Etidronate is a bisphosphonate that is known to prevent vascular calcification to a greater extent than other agents in this drug class, and has been shown to induce plaque regression when used in combination with atorvastatin. Kawahara and colleagues investigated whether etidronate has an individual effect, or whether its action is synergistic with that of statin therapy.

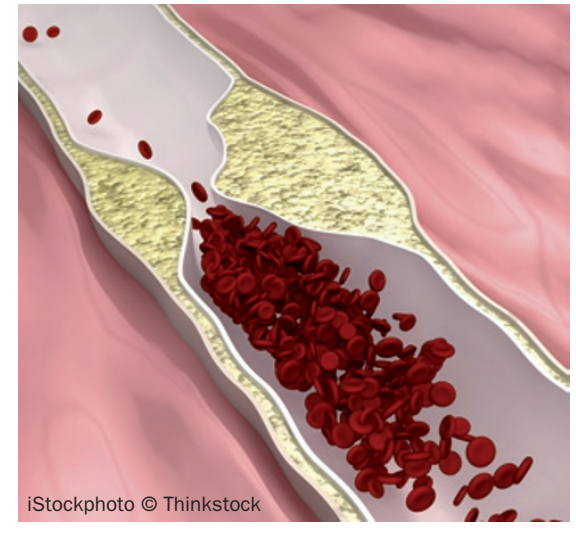

The investigators enrolled 108 patients with hypercholesterolaemia from medical centres in Niigata Prefecture, Japan. Participants were randomly assigned to receive atorvastatin $20 \mathrm{mg}$ per day for 1 year, etidronate $400 \mathrm{mg}$ per day for 14 days (repeated every 3 months for 1 year), or a combination of these therapies. The primary end point was 1 year change in maximal vessel wall thickness on MRI-a measure of plaque size, indicating therapeutic efficacy.
In the thoracic aorta, maximal vessel wall thickness was reduced by $13.8 \%$ and $12.3 \%$ in the combination therapy and atorvastatin groups, respectively, but was increased by $2.2 \%$ in the etidronate group (combination vs etidronate $P<0.001$ ). By contrast, in the abdominal aorta, maximal vessel wall thickness was reduced by $11.4 \%$ and $5.5 \%$ in the combination therapy and etidronate groups, respectively $(P=0.006)$. With atorvastatin monotherapy, the abdominal aortic maximal vessel wall thickness increased by $0.9 \%(P<0.001$ for combination vs atorvastatin). Dual therapy was, therefore, significantly more efficacious in both aortic segments than either agent alone. The investigators now plan to conduct a large trial to assess whether these morphological changes translate into reductions in clinical events.

\section{Alexandra King}

Original article Kawahara, T. et al. Atorvastatin, etidronate or both in patients at high risk for atherosclerotic aortic plaques: a randomized controlled trial. Circulation doi:10.1161/CIRCULATIONAHA.113.001534 